\title{
Application Analysis on Zhuzhe Hotel Management System in Chinese Hotel Chain
}

\author{
Yun Song ${ }^{1, a}$, Hairong Zeng', and Yanmei Meng ${ }^{3, c}$ \\ Qingdao Huanghai University, Qingdao, Shangdong, China \\ a155152036@qq.com, b24176040@qq.com, '35918721@qq.com
}

Keywords: Zhuzhe hotel management system, Hotel chain, Application research

\begin{abstract}
Due to the advanced hotel management system, the Chinese hotel chains will efficiently manage a large number of information and introduce the advanced management concept to improve the efficiency and service quality. Only when the hotel improve the internal management system constantly, it could improve the level of the hotel's decision, economic interests and the relationship benefits, and finally enhance competitiveness. Zhuzhe hotel management system is a free hotel management system developed under the condition of the hotel informatization. This article mainly analyzed the application of Zhuzhe hotel management system in small and medium-sized hotels. And through analysis, advantages and disadvantages are analyzed and evaluated. It could provide some guidance in the improvement and better application of the system.
\end{abstract}

\section{Introduction}

With the continuous development of our economy and the improving of people's living standard, tourism has become a new fashion. The rapid development of tourism industry directly led to the hotel sharp increase especially in the hotel chain, as well as the increasingly fierce competition between the hotels. To win in the competition, hotel chain must be better and more original in the service, product, management and operation. Hotel management directly influences its operating conditions. e China hotel chains, such as Huazhu and Home Inns have developed its own hotel management system, for developing your own system can be combined with its own characteristics and can bind the APP. In order to adapt to the challenges and opportunities of the information age, make good their own hardware and software at the same time, managers of budget hotel chains have introduced the hotel management system to improve the level of hotel management.

Therefore, hotel management information system, the necessary scientific tool, has become an important content of modern scientific management. After development of 20 years, hotel management information system has now reached a certain level, especially in recent years the hotel computer application demand. Under this background, the Beijing Zhuzhe Information Technology comprehensive put forward a set of chain hotel management system that firstly use JAVA language development, domestic pure B/S architecture based on web type. This system was combined with the advanced information systems of hotel chains at home and abroad. It also introduced the essence of the large Banks, telecommunications system used at home and abroad to the hotel management system. The benefits of the system are to make type chain hotel management system to be more rigorous, more accurate in calculation, more stable in performance, stronger in scalability. The system may include all kinds of existing hotel software system, reduce the input cost of hotel software and hardware, greatly reduce the cost for hotel management and create higher benefit for the hotel.

\section{Introduction of ZhuZhe hotel management system}

Zhuzhe international chain hotel management system, with the national star hotel standard business design, adapt advanced hotel management mode to fit the practical characteristics of fusion of Chinese hotel management. This system adopted the advanced domestic and international hotel 
management experience, integrating into the scientific, standardized, successful international hotel modern management thoughts. Combined with advanced computer technology, the management system can guarantee the safe and reliable information. It used the most advanced $\mathrm{B} / \mathrm{S}$ architecture, the software includes: front desk management, Internet information management, the background information, and used stable SQLserver2000 database, the Client/Server and Browser/Server architecture.

It is a type of date software of management the data from the server to the client. All menus, window, operating mode of the software are the browser style. Hotel management involved in the project, can be seen directly in the interface. It's very convenient. Password management can ensure the security of the software. Other functions such as database backup, restore, initialization and encryption can ensure data security. System built-in logging that record all the computer operation will ensure that the guest information changes to the documented. This software is a security, stable, fast operation of hotel management software based on the powerful way of fuzzy query and report query, powerful online help software.

\section{The constitute of Zhuzhe hotel management system}

Zhuzhe hotel management system integrated with advanced management experience and information systems of global leading hotel chains, such as 7 days, home inns chain. It adapt the three-tier architecture based on J2EE and lightweight structure to form the formation of modular software structure and plug-in architecture. The AJAX technology can improve the user experience and increase the manipulation of the system. The agile development can guarantee the quality of the products and ensure progress.

\section{B/S architecture based on J2EE}

$\mathrm{B} / \mathrm{S}$ is the abbreviation of Browser/Server (Browser/Server). B/S is the technique supported by Microsoft, IBM, HP, and Lenovo, Founder, TongFang, Thunis. The B/S structure management software dominates the global mainstream and the dominant position in the field of management software. At present most domestic hotel management system still adapt $\mathrm{C} / \mathrm{S}$ architecture that is relatively backward.

Zhuzhe hotel management system is developed through the JAVA language, based on Web2.0 pure $\mathrm{B} / \mathrm{S}$ structure of hotel management system. While some other system on the market use .Net language development of $\mathrm{B} / \mathrm{S}$ architecture or adopt $\mathrm{B} / \mathrm{S}$ combining with traditional $\mathrm{C} / \mathrm{S}$ system of hotel management.

$\mathrm{B} / \mathrm{S}$ structure can be installed within a local area network (LAN) and can be based on wide area network. It's no need to install the client, as long as the operating system and browser can be used commonly. It is quite easy to install, upgrade and maintain. Users can update by downloading and installing it on oneself other than tedious operation in client and server. Thus the boss can see a report on the net at home while drinking coffee and listening to music,

\section{The multi-tier architecture}

The multi-tier architecture includes data access layer, business logic layer, display layer and external interface layer. The multi-tier architecture are of great flexibility. Any changes in the other levels won't affect the other layers.

\section{The modular software structure}

The structure is a group of module which abstracted out the central reservation system, member management system, the central management system and the public portion of the business logic of the branch management system. These components can be shared by big system and be assembled the new functionality.

\section{The plug-in architecture}

Plug-in architecture, which makes the product has a great deal of flexibility, increases the support a database or WAP website, without breaking the original program. The system can be run steadily 
just by writing a plug-in plus a support part of the code. This greatly increases the stability of the product, speed of the new version and the new module is introduced.

\section{Ajax-based Web applications}

The response speed is the biggest benefit of local area network (LAN), while other maintenance, installation simplicity and convenience are far less than web applications. With AJAX, web applications will not only respond to user input, but respond to every page and every page items instantly, such as input boxes, buttons, drop-down choice. It also can update only a fraction of any page. The structure of the application has brought the revolutionary change. A large number of $\mathrm{C} / \mathrm{S}$ will be transplanted to $\mathrm{B} / \mathrm{S}$ structure of the program transplantation. Representative application, such as Gmail (Google's email, Google's word processing system and form processing system - the online Office) based on AJAX Web applications, not only increase the user experience, and greatly enhances the ability of concurrent access.

\section{Agile development model to ensure the quality}

Agile development mode is popular in the IT industry development model in Europe. It is put forward against large unified software development process (RUP) defects - interim design, documentation, no guarantee that high quality product delivered on time. It combined with test driven development, pair programming, encouraging communication, continuous integration, automated testing principles. It can effectively control the project risk and ensure that high quality and low defect, on time delivery of the software and project development. The development process of Zhuzhe hotel management system just follow the agile development to ensure the high quality requirements.

\section{The Advantage of Zhuzhe Hotel Management System}

\section{Convenient management with unlimited sites}

Access to the hotel, anytime and anywhere. The most advanced B/S technology is used, and hotel managers, whether at home or away on a business trip, as long as they can get to the Internet, they can refer to the hotel operating conditions and take control of any abnormal operation at any time.

Having hotel management software that is freed from VPN completely, which can save much work and the costs of purchasing hardware and software, as well as the annually VPN maintenance fees. Remote inquiry and management no longer need to purchase and bind the VPN.

Pure B/S technology with unlimited sites. Within one hotel, the hotel owner can set up a lot of client-sides, thus saving the hotel fees and supporting the quick developing needs of the hotel. However, the traditional C/S hotel management software is charged according to the sites.

Beyond the $\mathrm{C} / \mathrm{S}$ technology in an all-round way. The management system of Zhuzhe hotel is installed in the hotel's server (host). When the hotel managers don't want to use the network, it is a local area network (LAN), and the hotel can work normally with unlimited sites. When the hotel is connected to the network, it is very convenient for the hotel owner to search the hotel real-time operating conditions anytime anywhere.

\section{Fast check-in with no wait}

The application of Zhuzhe hotel management system is stored in the server. All applications update with the server, and there is no need to install tedious installation programs such as the client-side; With the center database, only backuping and maintaining a database could make the hotel quickly respond to the hotel business process improvement and re-engineering. With such hotel management system, fast check-in can be realized only by inputting few necessary information such as check-in and check-out dates. Fast check-in can be realized within only three to five seconds, allowing no waiting for the guests, and the hotel receptionist can input the guests' information and deposit information when he or she is not busy.

\section{Stereoscopic and Safe Protection Measures}

Relatively, other software manufacturers can only adopt the Windows System. Zhuzhe Hotel Management System, based on the development of Java language crossing platform, can be smooth to the Unix platform. Linux/Unix system is a operating system widely used by the large sites and banks, telecommunication systems in the world. It is the only one without virus, and hard to be 
attacked by hackers. Today, the hardware firewalls are mostly developed and made by using the Linux/Unix System. Zhuzhe Hotel Management System adopting the Linux operating system brings absolute security to the small and medium-sized hotels. It is also the escort of the small and medium-sized hotels' management. Pure B/S structure ensures the safety in the process of data transmission.

The safe access to login and visit is realized by using multi-stage role management; The internal and external networks can be isolated physically and allowed to the authorized computer using; The external net access is transmitted by authentication and data encryption, which makes managers truly safe.

\section{Saving the Charges of Software and Hardware}

Zhuzhe Hotel Chain Management System is pure B/S, not VPN. Each branch could save the cost of hardware VPN for thousands of yuan.

Zhuzhe Hotel Management System (PMS) is also based on B/S, which can be visited as long as you have a browser; The promotion server is used for upgrading, so that the system maintenance overhead can be minimized and the IT maintenance personnel of chain hotel can be reduced by half. Meanwhile, with a unique \"digital $\backslash$ function, Zhuzhe central journaling greatly saves time spending on the financial personnel auditting account, which makes it possible that two branches can share a financial personnel and save a lot of labor costs.

The Web Services middleware developed by Beijing Zhuzhe integrate seamlessly various systems in the hotel(including OA, ERP, financial system, foreground management system, member management system, the central reservation system, customer relationship management system, etc.), achieving the integration of hotel resources, saving a lot of costs of software procurement for hotel at the same time.

Beijing Zhuzhe is the first domestic B/S hotel managing system to adopt the Postgresql MySQL, which is not a piratic database but completely free. It can save the purchasing cost of legitimate commercial database for tens of thousands yuan or even hundreds of millions yuan.

\section{Perfect Realization to the Butt-Joint Headquarters and Branch}

Since Zhuzhe Hotel Chain Managing system adopts pure B/S, without VPN, with fast speed, redundancy check, and failure retransmission mechanism, which can guarantee reservation and state from losing and realize perfectly the docking of branches and headquarters. However, because of other $\mathrm{C} / \mathrm{S}$ architecture firms using VPN to butt each branch, not only is its speed of transmission quite slow, but also it leads to data loss. Transmission speed is slow and reservation information is often lost when the number of branches exceeds five. When exceeding ten, the summary report of the headquarters may be slower. While exceeding twenty, the speed of headquarters' summary data is unbearable and data loss is over $20 \%$. Loyalty points on the product could not be often increased. This is because the effect VPN transmission speed and the two different structures' compatibility generating. Many hotel chains have switched $\mathrm{C} / \mathrm{S}$ to $\mathrm{B} / \mathrm{S}$ system completely, such as the Seven-day and the Home Inn Hotel Chain; After using C/S for many years, Jiangxi Life Modern Hotel has switched to $\mathrm{B} / \mathrm{S}$ as well. Beijing Zhuzhe adopts pure $\mathrm{B} / \mathrm{S}$ architecture JAVA language developing, whose headquarters' the central and branch system is $\mathrm{B} / \mathrm{S}$ structure. Currently, the pure $\mathrm{B} / \mathrm{S}$ architecture is very few. The head quarter and branch are docked with a good compatibility. VPN, the third party software, is not used for docking. The phenomenon of loss of data couldn't occur. When the Banks and other large systems are adopted to this model, supporting for thousands of stores, the operating speed will not be effected as long as you make sure the server group and bandwidth .

\section{Powerful functions of online marketing and booking}

Zhuzhe Hotel Management System, this system possesses these functions such as weibo, SMS marketing and so on. The website hits and the degree of activity can be improved by interacting with fans and proceeding a variety of promotions through the hotel official weibo. Downloading the mobile client, map search and positioning by mobile phones, the clients feel free to book the hotels around. The reservation and query orders via mobiles phones, these just cater to the habits of the young people's Internet booking . 
This system can dock well with Zhuzhe online booking system, clients can book hotel rooms in the portal sites online, which is the real meaning of real-time online booking. After landing the hotel online booking system through the clients' membership number, ID number and cell phone number, the system can send the booking information to the hotel front desk management system automatically, then this system will set aside the reservation, and this process needn't the front desk staff manual operation, which realizes the booking online.

\section{Convenient maintenance and 24 hours service}

The convenient and efficient upgrade and maintenance. Seamless upgrade, users can download, install and upgrade it, which doesn't need each client and server to upgrade the cumbersome process operations any more. B/S architecture can be upgraded and maintained at any time, which does not delay the normal operation of the hotel.

Service 24 hours a day. The Zhuzhe hotel management system in Beijing provides $7 * 24$ hours' service for the customers, and protect the interests of customers at maximum. At the same time it can provide the 10-minute quick response to maintenance .

\section{Conclusion}

Zhuzhe hotel management system has been successfully adopted by some well-known hotel chains at home and abroad such as Beijing Yimeijia Convenient Hotel Chain Co., LTD., Jiangxi Yongsheng Moderns Hotel Chain, Ningxia Lanhuahua Hotel Chain, Beijing Hualijiahe Hotel Chain and so on, running in good condition. Admittedly, in this system, there are certain inadequacies because the software developers are not the hotel staff and they always want to prepare for everything during the development of the system, no focus, more functions but a bit of a mess and some of them are useless for these hotel chains; No implanted radio frequency technology, which enable customers' identities will be confirmed and recognized within a short distance as long as they carry an embedded RFID card which use radio to answer the code readers and the relevant information of the users is stored in the chip, each tag with a unique ID number, connecting to the related billing database for being processed.

At present, Zhuzhe hotel management system has been widely used by the hotel chains in our country as well as some small and medium-sized hotels abroad. Its value is definite. The hotel industry has been reforming and developing continuously in our country, for example, in recent years the mid-priced hotels have been exploding with a bright future, and Zhuzhe hotel management system can also be developed in this direction.

\section{Acknowledgement}

Innovation and Entrepreneurship Education Project in Qingdao Huanghai University(Project Number:2016cxcy1)

\section{Reference}

[1]Tan Wenhui. Research of Realization of the Information Management of the Hotel Industry [J]. Journal of Chinese business, 2010 (10)

[2]Wang Zhe. Talking about information construction of our country economy hotel [J]. Journal of hospitality management research, 2014 (9)

[3]Wang Qing. Research of Application of Management Information System in Hotel Management [J]. Science and technology innovation herald, 2008 (13)

[4]Yang Huihui , Deng Wei, Chen Chong. Application of Big Data in Hotel Management System[J]. Science and technology plaza, 2015 (12) 